\title{
Classificação de clones de Eucalyptus por meio da relação siringil/guaiacil e das características de crescimento para uso energético
}

\author{
Classification of Eucalyptus clones by the ratio syringyl/guaiacyl \\ and growth characteristics for energy use
}

\author{
Thiago de Paula Protásio1, Paulo Fernando Trugilho², Ana Clara Caxito de Araújo ${ }^{3}$, \\ Thomaz Aurélio Bastos ${ }^{4}$, Sebastião Carlos da Silva Rosado ${ }^{2}$ e Jefferson Fernando Naves Pinto ${ }^{5}$
}

\begin{abstract}
Resumo
O objetivo deste trabalho foi verificar a viabilidade de uso da relação siringil/guaiacil da lignina e da produtividade de massa seca na classificação de clones de Eucalyptus visando à geração de calor e a produção de carvão vegetal, empregando-se técnicas univariadas e multivariadas. Foram avaliados 39 clones de Eucalyptus spp., aos 81 meses de idade, sendo amostradas três árvores por material genético. Todas as árvores foram cubadas rigorosamente para a obtenção do volume individual. Para a classificação e agrupamento dos clones de Eucalyptus foram utilizadas as seguintes características: densidade básica, teor de lignina total, relação siringil/guaiacil (S/G), volume individual sem casca e as estimativas de massas secas de madeira, de carbono e de lignina total. Procedeu-se às análises de variância univariadas e para o agrupamento dos clones utilizou-se o algoritmo de Scott-Knott a $5 \%$ de significância. Utilizou-se, ainda, a análise multivariada de agrupamento (cluster) visando obter grupos de clones similares. Observou-se que as estimativas de massa seca de madeira e de carbono foram as variáveis que mais contribuíram para a divergência genética total, sendo decisivas no agrupamento e classificação dos clones de Eucalyptus para a finalidade energética. $O$ teor de lignina total, a relação $S / G$ e a estimativa de massa de lignina contribuíram pouco para a diversidade genética dos materiais analisados, porém não devem ser negligenciadas no ranqueamento de clones para a produção de carvão vegetal ou energia térmica.
\end{abstract}

Palavras-chave: divergência genética, agrupamento, qualidade da madeira, lignina.

\begin{abstract}
The objective of this study was to determine the feasibility of using the ratio syringyl/guaiacyl of lignin and dry matter productivity in the classification of Eucalyptus clones, in order to generate heat and for charcoal production, using univariate and multivariate techniques. 39 Eucalyptus spp. clones were evaluated at 81 months of age, and three trees were sampled per genetic material. All trees were accurately scaled for obtaining of the individual volume. For the classification and grouping of Eucalyptus clones, the following characteristics were used: basic density, total lignin content, ratio syringyl/guaiacyl (S/G), individual volume under bark and estimates of wood dry matter, carbon and total lignin. Univariate analyses of variance were performed and, for clone grouping, the Scott-Knott algorithm at $5 \%$ significance was used. Multivariate cluster analysis was also used, in order to obtain groups of similar clones. It was observed that wood dry matter and carbon estimates were the variables that most contributed to the total genetic divergence, being decisive in the grouping and classification of Eucalyptus clones for energy purposes. The content of total lignin, ratio S/G and matter estimate of lignin little contributed to the genetic diversity of the analyzed materials. However, they should not be overlooked in clone ranking for charcoal or thermal energy production.
\end{abstract}

Keywords: genetic divergence, grouping, wood quality, lignin.

\footnotetext{
1Professor Adjunto do Curso de Engenharia Florestal. Universidade Federal Rural da Amazônia, Câmpus de Parauapebas, PA 275, Km 13, Zona Rural, Caixa Postal 3017, Parauapebas, PA, Brasil. E-mail: depaulaprotasio@gmail.com

2Professor Titular do Departamento de Ciências Florestais. UFLA - Universidade Federal de Lavras. Caixa Postal 3037 37200-000, Lavras, Brasil. E-mail: trugilho@dcf.ufla.br; scrosado@ufla.br

32Doutorando em Ciência e Tecnologia da Madeira. UFLA - Universidade Federal de Lavras. Caixa Postal 3037 - $37200-000$, Lavras, Brasil. E-mail: anacaxitoengflor@hotmail.com

${ }^{4}$ Graduando em Engenharia Florestal. UFLA - Universidade Federal de Lavras. Caixa Postal 3037 - 37200-000, Lavras, Brasil. E-mail: thomazz2@hotmail.com

${ }^{5}$ Mestre em Agronomia / Produção Vegetal. UFGO - Universidade Federal de Goiás / Regional Jataí - Câmpus Cidade Universitária. Caixa Postal 3 - Rodovia BR-364, km 192 - Parque Industrial - 75801615 - Jataí, GO, Brasil. E-mail: jeffnaves@gmail.com
}

Sci. For., Piracicaba, v. 45, n. 114, p. 327-341, jun. 2017 DOI: dx.doi.org/10.18671/scifor.v45n114.09 


\section{INTRODUÇÃO}

Um dos problemas ambientais mais importantes refere-se às elevadas emissões de gases causadores de efeito estufa predominantemente devido ao uso de fontes fósseis, especialmente o petróleo, carvão mineral e gás natural, como a principal forma de se obter calor e eletricidade em diversos países no mundo (MUNIR et al., 2009; SHEN et al., 2010). Para resolver essa situação muitas nações têm utilizado fontes alternativas de energia em substituição aos combustíveis não renováveis, tais como a madeira ou outras biomassas lignocelulósicas (YIN, 2011).

Nesse sentido, o Brasil se destaca por apresentar uma matriz energética com 39,4\% da oferta interna de energia proveniente de fontes renováveis, em que a lenha e o carvão vegetal representam 8,1\% do total (EPE, 2015). No país, em 2014, foram consumidos 79.768 .000 toneladas de lenha, sendo que 34,2\% foram destinados à produção de carvão vegetal e geração elétrica (EPE, 2015). Segundo a Indústria Brasileira de Árvores (IBÁ,2015), em 2014, foram consumidos 5.300.000 toneladas de carvão vegetal, com $81 \%$ de participação de madeira oriunda de árvores plantadas, principalmente clones e espécies de Eucalyptus.

Diante importância da lenha e do carvão vegetal para o Brasil, há a necessidade de estudos capazes de disponibilizar para o setor florestal brasileiro materiais genéticos de Eucalyptus que conciliem características de crescimento e de qualidade da madeira que poderão otimizar a produtividade energética por área de plantio.

Nos últimos anos, pesquisas têm sido realizadas com o objetivo de avaliar os efeitos de clone ou espécie (CARNEIRO et al., 2014; PEREIRA et al., 2012; PEREIRA et al., 2013); idade (CARNEIRO et al., 2014; NEVES et al., 2013; SANTANA et al., 2012); de sítio ou local de plantio (MENESES et al., 2015; NEVES et al., 2011); de espaçamento (REIS et al., 2012); de tratos silviculturais (OLIVEIRA et al., 2012) e de posições na árvore (ARANTES et al., 2011; 2013) na qualidade da madeira para a geração direta de calor ou para a produção de carvão vegetal. Estes estudos foram relevantes, pois tentaram estabelecer relações de influência das propriedades físicas, químicas, anatômicas e mecânicas da madeira na produtividade, qualidade e desempenho do carvão vegetal nos equipamentos industriais de conversão energética. Entretanto, ainda existem lacunas que devem ser consideradas.

Dessa forma, para que se tenha produtividade aliada à qualidade da madeira é importante investir em um programa de seleção de materiais genéticos que apresentem características da madeira mais adequadas à produção de energia e de carvão vegetal, tais como elevada densidade básica, alto teor de lignina, baixo teor de holocelulose, baixo teor de minerais e fibras de parede celular mais espessa.

Além destas características, tem-se destacado nas pesquisas mais recentes a importância de se avaliar a composição ou qualidade da lignina da madeira de Eucalyptus, ou seja, a relação siringil/ guaiacil (ARAÚJO et al., 2016; CASTRO et al., 2016; 2013; GOUVÊA et al., 2015; PEREIRA et al., 2013; SANTOS et al., 2016; SOARES et al., 2014). Segundo Sarkanen e Ludwig (1971), a lignina é uma macromolécula formada a partir da polimerização desidrogenativa enzimática de três álcoois precursores: o trans-p-cumárico (unidade cumaril), trans-coniferílico (unidade guaiacil) e trans-sinapílico (unidade siringil).

Espera-se que quanto menor a relação siringil/guaiacil (S/G) maior será o rendimento gravimétrico em carvão vegetal em decorrência da maior condensação da macromolécula de lignina e, consequentemente, da sua maior resistência à degradação térmica (CASTRO et al., 2013; GOUVÊA et al., 2015; PEREIRA et al., 2013). O poder calorífico da madeira também pode ser influenciado pela relação S/G. Sabe-se que a unidade guaiacil apresenta maior relação $\mathrm{C} / \mathrm{O}$, comparativamente à unidade siringil (SOARES et al., 2014), e isso aumenta o valor calórico, pois o carbono é o principal elemento químico combustível.

Apesar de Castro et al. (2013) e Pereira et al. (2013) utilizarem a relação siringil/guaiacil da lignina como parâmetro de classificação de clones de Eucalyptus para a finalidade energética, ainda existe carência de pesquisas relacionadas a aplicação desse índice, juntamente com as características de crescimento, especialmente na avaliação de materiais clonais.

Dessa forma, o objetivo deste trabalho foi verificar a viabilidade de uso da relação siringil/ guaiacil da lignina e da produtividade de massa seca na classificação de materiais genéticos de Eucalyptus visando à geração de calor e a produção de carvão vegetal, empregando-se técnicas univariadas e multivariadas. 


\section{MATERIAL E MÉTODOS}

\section{Materiais biológicos, amostragem e cubagem das árvores}

Neste trabalho, foram avaliados 39 materiais de Eucalyptus spp., aos 81 meses de idade. O material biológico foi proveniente de teste clonal, instalado em espaçamento $3 \mathrm{~m} \mathrm{x} 3 \mathrm{~m}$, da empresa Plantar, localizado no município de Curvelo, estado de Minas Gerais (18 ${ }^{\circ} 45^{\prime} 06.44^{\prime \prime}$ S; 44 $33^{\prime} 39.16^{\prime \prime} \mathrm{O}$ e 690 $\mathrm{m}$ de altitude), como apresentado na Tabela 1. Foram amostradas três árvores de diâmetro médio por material genético. Nas árvores, foram retirados discos de 2,5 cm de espessura em cinco pontos da altura comercial do tronco, ou seja, na base, a 2, 10, 30, 50 e 75\% da altura comercial da árvore, considerada até um diâmetro mínimo de 4,0 cm com casca, conforme recomendações de Downes et al. (1997).

As árvores foram cubadas nas posições fixas de 0,10 m, 0,70 m, 1,30 m, 2,0 m, e a partir desta, a cada 2 metros até a altura comercial. Posteriormente, o volume individual com e sem casca foi estimado utilizando-se a equação de Smalian.

Tabela 1. Relação dos materiais genéticos avaliados, altura comercial (Hc), diâmetro a altura do peito com (DAPcc) e sem casca (DAPsc).

Table 1. List of genetic materials evaluated, commercial height (Hc), diameter at breast height with (DAPcc) and without bark (DAPSC).

\begin{tabular}{|c|c|c|c|c|c|}
\hline $\mathbf{N}^{\circ}$ & Clone & Espécie/Híbrido & $\mathrm{Hc}(\mathrm{m})$ & DAPcc (cm) & DAPsc (cm) \\
\hline 1 & 1001 & E. urophylla & 18,73 & 14,49 & 13,36 \\
\hline 2 & 1002 & Eucalyptus sp. & 23,23 & 15,93 & 14,98 \\
\hline 3 & 1003 & E. urophylla & 21,63 & 15,30 & 14,35 \\
\hline 4 & 1004 & E. urophylla $\times$ E. camaldulensis & 21,73 & 15,12 & 14,31 \\
\hline 5 & 1005 & E. urophylla & 21,03 & 15,80 & 14,78 \\
\hline 6 & 1006 & E. urophylla & 23,28 & 17,24 & 16,21 \\
\hline 7 & 1007 & E. urophylla & 23,03 & 16,64 & 15,62 \\
\hline 8 & 1008 & E. urophylla & 20,83 & 16,56 & 15,83 \\
\hline 9 & 1009 & E. urophylla & 21,47 & 15,50 & 14,31 \\
\hline 10 & 1010 & E. urophylla & 21,82 & 16,68 & 15,59 \\
\hline 11 & 1011 & E. urophylla & 19,47 & 14,93 & 13,95 \\
\hline 12 & 1012 & E. urophylla $\times$ E. grandis & 21,37 & 14,06 & 13,33 \\
\hline 13 & 1013 & E. urophylla $\times$ E. grandis & 20,03 & 15,41 & 14,31 \\
\hline 14 & 1014 & E. grandis & 19,82 & 14,35 & 13,14 \\
\hline 15 & 1015 & E. urophylla & 21,43 & 14,98 & 13,99 \\
\hline 16 & 1016 & Eucalyptus sp. & 21,38 & 15,37 & 14,42 \\
\hline 17 & 1017 & Eucalyptus sp. & 21,60 & 14,64 & 13,54 \\
\hline 18 & 1018 & Eucalyptus sp. & 22,27 & 16,85 & 15,77 \\
\hline 19 & 1019 & Eucalyptus sp. & 21,55 & 14,56 & 13,60 \\
\hline 20 & 1020 & Eucalyptus sp. & 19,35 & 15,53 & 14,71 \\
\hline 21 & 1021 & Eucalyptus sp. & 19,75 & 15,50 & 14,37 \\
\hline 22 & 1022 & E. urophylla $\times$ E. grandis & 20,60 & 14,16 & 13,17 \\
\hline 23 & 1023 & Híbrido de E. urophylla & 20,23 & 13,93 & 13,15 \\
\hline 24 & 1024 & Híbrido de E. urophylla & 20,40 & 14,65 & 13,69 \\
\hline 25 & 1025 & Híbrido de E. camaldulensis & 19,95 & 14,09 & 12,90 \\
\hline 26 & 1026 & E. urophylla $\times$ E. grandis & 20,13 & 16,24 & 15,10 \\
\hline 27 & 1027 & E. urophylla $\times$ E. grandis & 20,73 & 16,47 & 15,20 \\
\hline 28 & 1028 & E. pellita $\times$ E. grandis & 18,02 & 15,02 & 14,28 \\
\hline 29 & 1029 & E. urophylla $\times$ E. grandis & 20,33 & 15,44 & 14,70 \\
\hline 30 & 1030 & Eucalyptus sp. & 22,77 & 15,24 & 14,60 \\
\hline 31 & 1031 & Eucalyptus sp. & 23,67 & 17,06 & 16,05 \\
\hline 32 & 1032 & Eucalyptus sp. & 20,27 & 15,44 & 14,63 \\
\hline 33 & 1033 & Híbrido de E. urophylla & 22,72 & 15,46 & 14,59 \\
\hline 34 & 1034 & E. urophylla & 23,85 & 16,81 & 15,89 \\
\hline 35 & 1035 & E. urophylla & 22,25 & 15,79 & 15,10 \\
\hline 36 & 1036 & E. urophylla & 20,53 & 14,03 & 12,79 \\
\hline 37 & 1037 & Eucalyptus sp. & 21,65 & 19,55 & 18,45 \\
\hline 38 & 1038 & E. urophylla & 21,43 & 15,48 & 14,50 \\
\hline 39 & 1039 & Híbrido de E. grandis & 19,22 & 15,68 & 14,46 \\
\hline
\end{tabular}

$\mathrm{Hc}=$ altura comercial, DAPcc e DAPsc = diâmetros com e sem casca na altura do peito. 
Para a classificação e agrupamento dos clones de Eucalyptus foram utilizadas as seguintes características: densidade básica, teor de lignina total, relação siringil/guaiacil da lignina, volume individual sem casca e as estimativas de massa seca de madeira, massa seca de carbono e massa seca de lignina.

\section{Determinação da densidade básica da madeira}

Os discos retirados das árvores foram seccionados em quatro cunhas passando pela medula. Duas menores cunhas opostas foram utilizadas na determinação da densidade básica da madeira, seguindo as recomendações da NBR 11941 da Associação Brasileira de Normas Técnicas (ABNT, 2003). A densidade básica média da árvore (DBm) foi considerada como sendo a média aritmética dos pontos de amostragem longitudinal no tronco das árvores. As outras duas cunhas opostas foram utilizadas para as demais análises na madeira. Para as análises químicas da madeira foi considerada uma amostra composta por todas as posições longitudinais de amostragem.

\section{Determinação dos teores de carbono e lignina na madeira}

Por meio da análise química elementar realizada em um analisador universal da marca Elemen$\operatorname{tar}$ (modelo Vario Micro Cube), foi possível a quantificação, base à massa seca de madeira, do teor de carbono (C). As amostras foram moídas e peneiradas, sendo utilizada para o ensaio a fração que ficou retida entre as peneiras de $0,250 \mathrm{~mm}$ e $0,074 \mathrm{~mm}$. O procedimento laboratorial adotado seguiu as especificações do fabricante do equipamento.

O teor de lignina insolúvel (Klason) foi obtido de acordo com a metodologia proposta Gomide e Demuner (1986) e o teor de lignina solúvel (Klason) em ácido sulfúrico foi determinado de acordo com o método de Goldschimid (1971). O teor de lignina total foi obtido pelo somatório dos teores de lignina solúvel e insolúvel.

\section{Determinação da relação siringil/guaiacil}

A degradação da lignina, para posterior determinação da relação $S / G$, foi realizada pelo método da oxidação alcalina da madeira com nitrobenzeno, seguida da cromatografia líquida de alta eficiência (CLAE), para quantificação dos seus derivados, conforme a metodologia descrita em Lin e Dence (1992).

\section{Estimativas de massa seca por árvore}

A estimativa de massa seca de madeira por indivíduo foi obtida multiplicando o volume individual da árvore pela densidade básica média (MENESES et al., 2015; TRUGILHO et al., 2015), enquanto que a estimativa de massa seca de carbono foi considerada como sendo o produto da massa seca de madeira pelo teor de carbono elementar (TRUGILHO et al., 2010). A massa seca estimada de lignina por árvore foi calculada multiplicando a massa seca de cada árvore-amostra e o teor de lignina total (BOTREL et al., 2010).

\section{Análises estatísticas: univariada e multivariada}

Foram realizadas análises de variância univariadas utilizando-se o delineamento inteiramente casualizado (DIC), para avaliar o efeito de clone, para as seguintes características: densidade básica, teor de lignina, relação siringil/guaiacil, volume individual da árvore e estimativas de massa seca.

De forma preliminar às análises de variância foram aplicados os testes de Levene, Shapiro-Wilk e Durbin-Watson, ambos a 5\% de significância, para verificar a homogeneidade de variância, a normalidade e a ausência de autocorrelação dos resíduos, respectivamente. Além disso, observou-se a dispersão gráfica dos resíduos do modelo estatístico utilizado. Não foram observados desvios em relação aos pressupostos clássicos das análises de variância, validando assim os resultados estatísticos obtidos.

Para a comparação múltipla das médias e o agrupamento univariado dos clones de Eucalyptus utilizou-se o teste Scott-Knott a 5\% de significância (SCOTT; KNOTT, 1974).

Além disso, os clones foram agrupados por meio da análise multivariada denominada cluster, que permite a obtenção de um dendrograma de similaridade. A matriz de distância genética entre os clones foi estimada por meio da distância generalizada de Mahalanobis $\left(\mathrm{D}^{2}\right)$, conforme sugerido por Cruz e Carneiro (2006). A distância de Mahalanobis executa um processo de padronização, compensa as correlações entre as variáveis e é uma alternativa a multicolinearidade (HAIR JÚNIOR. et al., 2009). 
Para a obtenção do dendrograma considerou-se o método da ligação média (UPGMA) como técnica hierárquica aglomerativa. Para a determinação do número ótimo de grupos no dendrograma foi considerado o método de Mojena (1977). Adotou-se k=1,25 como regra de parada na definição do número de grupos, de acordo com Milligan e Cooper (1985).

A consistência do dendrograma obtido foi avaliada por meio do coeficiente de correlação cofenética, que mede a correlação entre as distâncias recuperadas do dendrograma com a matriz de distâncias originais entre os clones de Eucalyptus, conforme proposto por Sokal e Rohlf (1962). A contribuição relativa de cada característica avaliada na divergência dos clones de Eucalyptus foi obtida pelo método de Singh (1981).

Após o agrupamento, as distâncias entre e dentro dos grupos foram computadas e foram realizadas análises de variância univariadas considerando como fator de variação os grupos formados e o delineamento inteiramente casualizado. As médias das características de cada material genético foram consideradas como repetições, conforme realizado por Alvares et al. (2012). Para a comparação múltipla das médias de cada grupo utilizou-se o teste Scott-Knott a 5\% de significância (SCOTT; KNOTT, 1974).

As análises estatísticas foram realizadas por meio dos programas R (R CORE TEAM, 2014) e Genes (CRUZ, 2013).

\section{RESULTADOS E DISCUSSÃO}

\section{Características da madeira}

Pelo Teste F observou-se efeito significativo de clone ( $\mathrm{p}$-valor $<0,05$ ) para todas as características avaliadas. Dessa forma, espera-se, para cada característica quantificada, que pelo menos um dos clones avaliados seja diferente dos demais e esse resultado indica que existe variabilidade e possibilita a seleção de materiais genéticos superiores para a geração direta de calor ou produção de carvão vegetal.

Para a densidade básica (Figura 1) pode-se observar significativa variabilidade entre os clones e a formação de cinco grupos distintos, sendo que os materiais genéticos 1025 (Híbrido de Eucalyptus camaldulensis), 1039 (Híbrido de Eucalyptus grandis), 1009 (Eucalyptus urophylla), 1004 (Eucalyptus urophylla x Eucalyptus camaldulensis) e 1012 (Eucalyptus urophylla $\mathrm{x}$ Eucalyptus grandis) apresentaram estatisticamente os valores mais elevados e, consequentemente, se destacaram como potenciais para a geração de energia térmica ou para a produção de carvão vegetal de uso doméstico ou siderúrgico. Espera-se que o carvão vegetal produzido a partir da madeira desses clones apresente maior densidade aparente, maior densidade energética, maior quantidade de carbono fixo por volume e melhores propriedades mecânicas, conforme relatado por Carneiro et al. (2014), Protásio et al. (2015) e Couto et al. (2015).

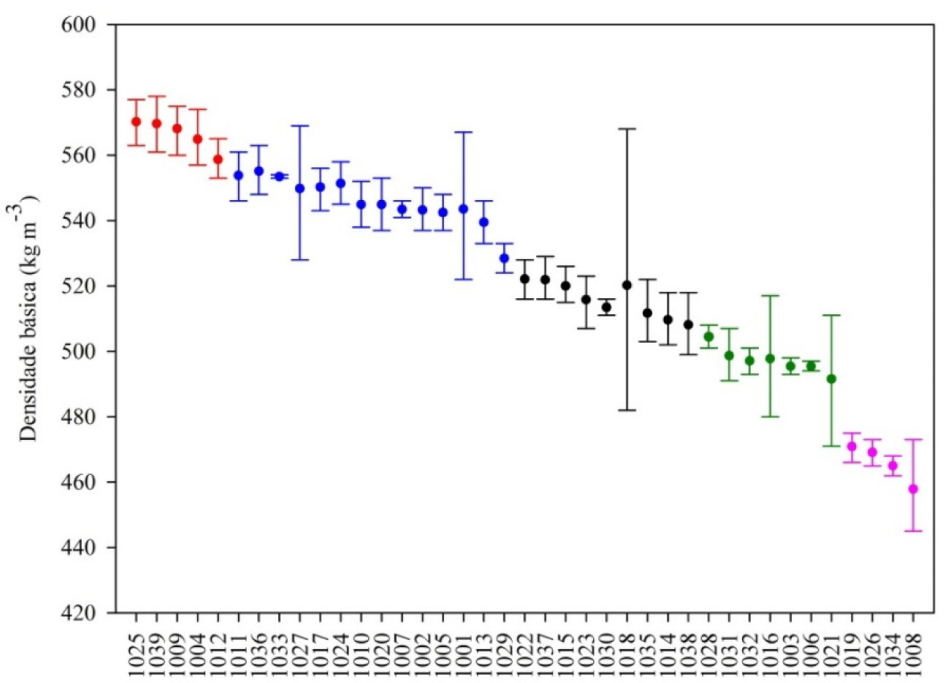

Figura 1. Densidade básica da madeira dos clones de Eucalyptus. As cores representam os grupos formados pelo teste de Scott-Knott $(a=0,05)$, as barras referem-se aos valores de máximo e mínimo e o coeficiente de variação experimental foi de $2,3 \%$.

Figure 1. Basic wood density of Eucalyptus clones. The colors represent the groups formed by the Scott-Knott test $(a=0.05)$, the bars refer to maximum and minimum values and the experimental coefficient of variation was $2.3 \%$. 
Carneiro et al. (2014), mencionaram ainda que, quanto maior a densidade da madeira, maior a quantidade de calor possível de ser liberada por metro cúbico. Os autores destacaram que essa característica é de grande relevância para a classificação de materiais genéticos para a queima direta da madeira, corroborando com os trabalhos de Protásio et al. (2013a, 2015).

Carneiro et al. (2014) e Castro et al. (2013) relataram densidade básica da madeira do clone GG 100, proveniente de um híbrido de Eucalyptus grandis $\mathrm{x}$ Eucalyptus urophylla, aos 84 meses, de $520 \mathrm{~kg}$ $\mathrm{m}^{-3}$. De forma semelhante, Soares et al. (2015) relataram valores de densidade básica da madeira de um híbrido de Eucalyptus grandis x Eucalyptus urophylla, com 84 meses, de $540 \mathrm{~kg} \mathrm{~m}^{-3}$. Esses valores reportados na literatura são semelhantes à média encontrada para o grupo de clones de Eucalyptus com maior densidade básica ( $566 \mathrm{~kg} \mathrm{~m}^{-3}$ ). Entretanto, para a confiabilidade da seleção de materiais genéticos destinados ao uso energético deve-se considerar ainda o teor de lignina total, a relação S/G e as produtividades de massa seca, pois esses parâmetros são desejáveis à produção de bioenergia.

Analisando-se a Figura 2, pode-se observar que para o teor de lignina os clones de Eucalyptus avaliados apresentaram menor variabilidade e, consequentemente, foram formados apenas três grupos de similaridade. Os grupos I, II e III apresentaram teores médios de lignina de 30,5\%, 29,2\% e 27,9\%, respectivamente. Vale salientar que os materiais genéticos avaliados são provenientes de seleções genéticas preliminares, sendo que isso justifica os maiores teores de lignina encontrados e a menor variabilidade para essa característica. Castro et al. (2013) e Protásio et al. (2013a) também relataram elevados valores médios de lignina total, superiores a 31\%, para clones de Eucalyptus destinados ao uso energético, assemelhando-se as tendências observadas no presente trabalho.

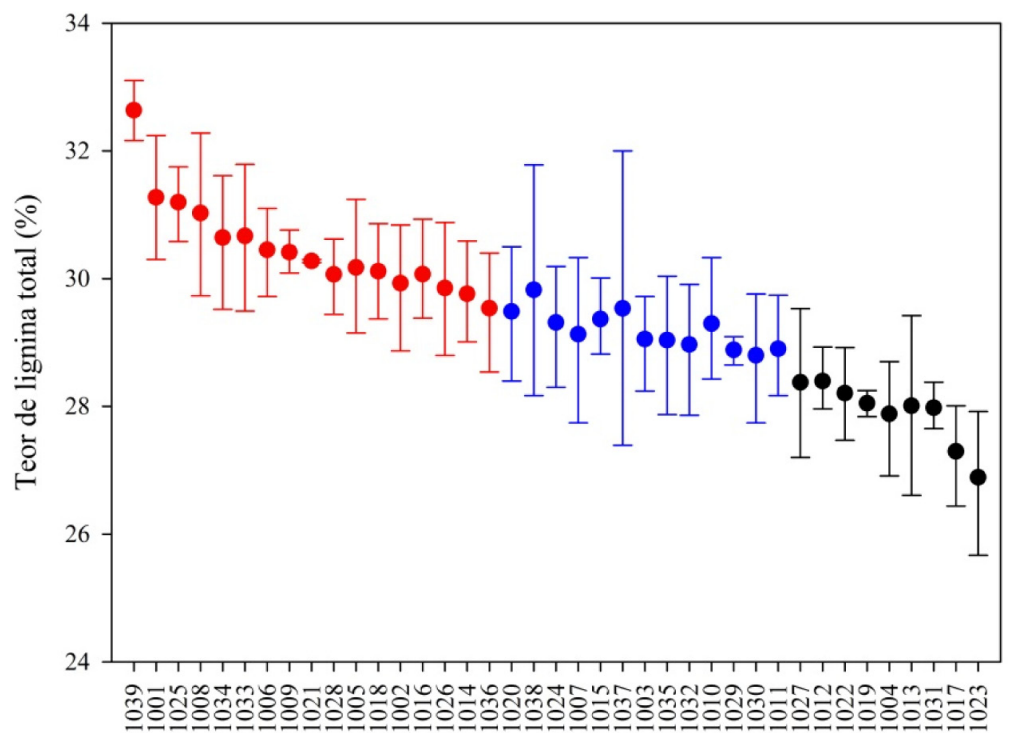

Figura 2. Teor de lignina total da madeira dos clones de Eucalyptus. As cores representam os grupos formados pelo teste de Scott-Knott $(a=0,05)$, as barras referem-se aos valores de máximo e mínimo e o coeficiente de variação experimental foi de 3,5\%.

Figure 2. Content of total lignin of Eucalyptus wood clones. The colors represent the groups formed by the Scott-Knott test $(a=0.05)$, the bars refer to maximum and minimum values and the experimental coefficient of variation was $3.5 \%$.

Observa-se que os clones 1039, 1025 e 1009, de maior densidade básica, encontram-se no grupo de maior teor de lignina total na madeira. Esse resultado reforça a possibilidade de uso desses materiais como fonte de biomassa para geração direta de energia ou produção de carvão vegetal. Dentre os componentes químicos moleculares a lignina é o que mais contribui para a formação do carvão vegetal, quando comparada à celulose e hemiceluloses, devido a sua elevada termorresistência atribuída, por sua vez, a sua estrutura química complexa e o seu alto grau de aromaticidade (RAAD et al., 2006; YANG et al., 2007). Além disso, madeiras com maior teor de lignina apresentam maior poder calorífico (TELMO; LOUSADA, 2011).

Contudo, alguns autores têm relatado a importância de avaliar, concomitantemente com o teor de lignina e demais índices de qualidade da madeira para geração de energia, a relação siringil/ guaiacil desta macromolécula (CASTRO et al., 2013; GOUVÊA et al., 2015; PEREIRA et al., 2013; PROTÁSIO et al., 2013b; SOARES et al., 2014; 2015). 
Observa-se, na Figura 3, que os clones 1016, 1022, 1031, 1021, 1039, 1025 e 1028, foram considerados estatisticamente similares para essa característica e formaram um único grupo com menor relação S/G (média de 2,6). Por outro lado, o grupo formado pelos clones 1024, 1035, 1023, 1038 e 1018, apresentou a maior relação siringil/guaiacil (média de 3,8).

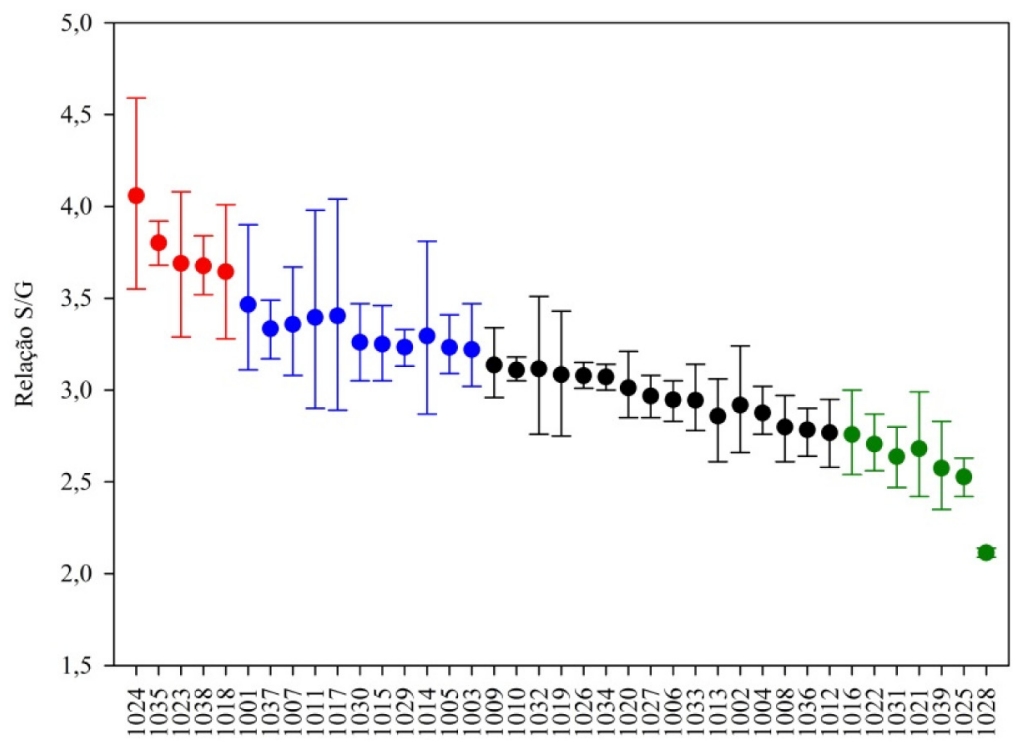

Figura 3. Relação siringil/guaiacil da lignina da madeira dos clones de Eucalyptus. As cores representam os grupos formados pelo teste de Scott-Knott $(a=0,05)$, as barras referem-se aos valores de máximo e mínimo e o coeficiente de variação experimental foi de $9,3 \%$.

Figure 3. Ratio syringyl/guaiacyl of lignin of Eucalyptus wood clones. The colors represent the groups formed by the Scott-Knott test $(a=0.05)$, the bars refer to maximum and minimum values and the experimental coefficient of variation was $9.3 \%$.

Provavelmente, quanto menor a relação S/G maior o poder calorífico da madeira, pois a unidade guaiacil apresenta na sua estrutura química proporcionalmente mais carbono que oxigênio comparativamente a unidade siringil (SOARES et al., 2014).

Além disso, Gouvêa et al. (2015), Pereira et al. (2013) e Soares et al. (2014, 2015) relataram que a menor relação $S / G$, ou maior proporção de unidades guaiacil, contribui para um maior rendimento gravimétrico em carvão vegetal. A unidade guaiacil apresenta um grupo metoxila $\left(-\mathrm{OCH}_{3}\right)$ a menos no anel aromático e isso possibilita a ocorrência ligações $\mathrm{C}=\mathrm{C}, \mathrm{C}-\mathrm{C}$ ou C-O-C entre os anéis aromáticos e, consequentemente, espera-se mais resistência térmica na madeira (PEREIRA et al., 2013).

Dessa forma, observa-se que para as características avaliadas na madeira os clones 1039 e 1025 se destacaram, pois apresentaram maiores valores de densidade básica da madeira e teor de lignina total e menores valores para a relação S/G. Assinala-se que as características de crescimento também devem ser consideradas na classificação de clones para a finalidade energética, conforme recomendação de Trugilho et al. (2001).

Quanto aos valores reportados na literatura, Pereira et al. (2013) observaram relação S/G média de 2,55 para dois clones de Eucalyptus camaldulensis, aos 90 meses de idade, corroborando com o encontrado para o clone 1025. Já Castro et al. (2013) relataram para clones comerciais de Eucalyptus grandis x Eucalyptus urophylla, Eucalyptus grandis e Eucalyptus camaldulensis, aos 84 meses de idade, valores para a relação $S / G$ de 2,40, 2,75 e 2,35, respectivamente. Esses valores assemelham-se ao obtido para os clones pertencentes aos grupos com menores relações $\mathrm{S} / \mathrm{G}$.

\section{Produtividade volumétrica e estimativas de massa seca}

Observa-se, na Figura 4, que o clone 1037 apresentou o maior volume individual sem casca e estatisticamente foi considerado um grupo dissimilar dos demais materiais genéticos avaliados. $\mathrm{O}$ teste de comparação múltipla utilizado permitiu o agrupamento dos clones em seis grupos evidenciando, assim, a variabilidade dos materiais genéticos de Eucalyptus para essa característica.

O clone 1037, grupo I, apresentou volume individual 103\% maior que o observado para os clones classificados no grupo VI de menor produtividade. Trugilho (2009) observou volume individual de árvores de espécies de Eucalyptus, aos 84 meses, variando de $0,0853 \mathrm{~m}^{3}$ a $0,4180 \mathrm{~m}^{3}$, ou seja, uma variação média de $390 \%$. Destaca-se que o autor analisou povoamentos comerciais oriundos de sementes e, por isso, a variabilidade encontrada para o volume das árvores foi superior ao relatado neste trabalho. 
Protásio et al. - Classificação de clones de Eucalyptus por meio da relação

Siringil/Guaiacil e das características de crescimento para uso energético

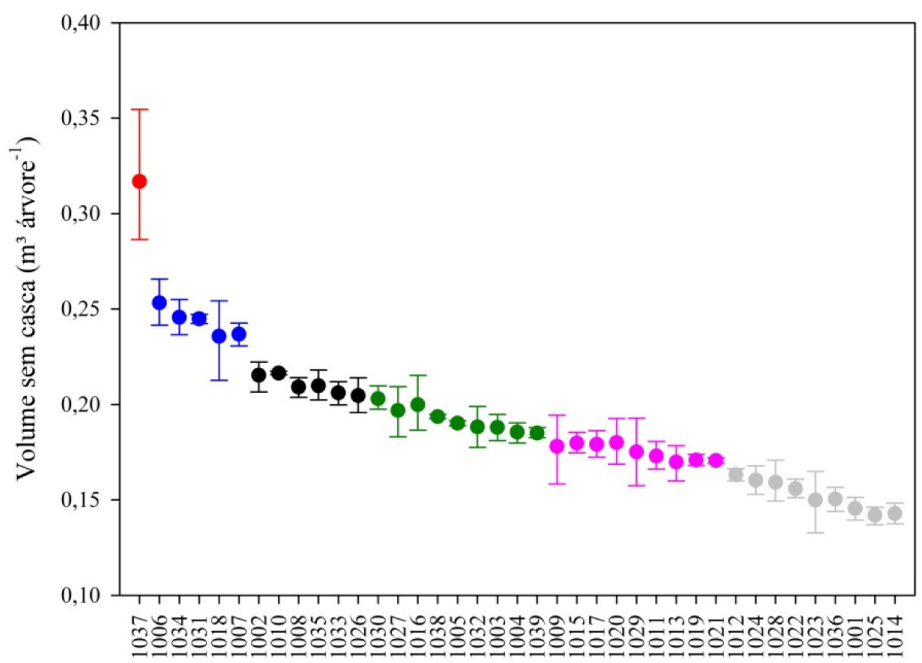

Figura 4. Volume individual sem casca das árvores dos clones de Eucalyptus. As cores representam os grupos formados pelo teste de Scott-Knott $(a=0,05)$, as barras referem-se aos valores de máximo e mínimo e o coeficiente de variação experimental foi de $6,0 \%$

Figure 4. Individual shelled volume of trees of Eucalyptus clones. The colors represent the groups formed by the Scott-Knott test $(a=0.05)$, the bars refer to maximum and minimum values and the experimental coefficient of variation was $6.0 \%$.

O material genético 1037, mesmo não apresentando destaque para as características da madeira, apresentou volume individual sem casca $117 \%$ maior ao observado para o clone 1025 e $68 \%$ maior em relação ao clone 1039 .

O clone 1025, apesar dos resultados favoráveis encontrados para a qualidade da madeira, apresentou baixa produtividade volumétrica e, consequentemente, foi classificado no grupo com as menores estimativas de massas secas de madeira, carbono e lignina total (Figuras 5, 6 e 7). Por outro lado, o clone 1039 pode ser considerado intermediário para essas mesmas características. Esses resultados desclassificam os clones 1025 e 1039 para a finalidade energética, pois quanto maiores às estimativas de massa seca maiores serão as produtividades de carvão vegetal e calor por área de plantio.

Conforme mencionado por Trugilho et al. (2001), o fator crescimento foi decisivo na classificação de clones de Eucalyptus para a finalidade energética, pois a produtividade de massa seca influenciou o ranqueamento dos clones, assemelhando-se ao observado neste trabalho. Meneses et al. (2015) e Trugilho (2009) também relataram que o volume individual sem casca interferiu na estimativa de massa seca de madeira. Dessa forma, materiais genéticos de baixa densidade básica, mas com elevada produtividade volumétrica se destacaram com as maiores estimativas de massa seca de madeira.

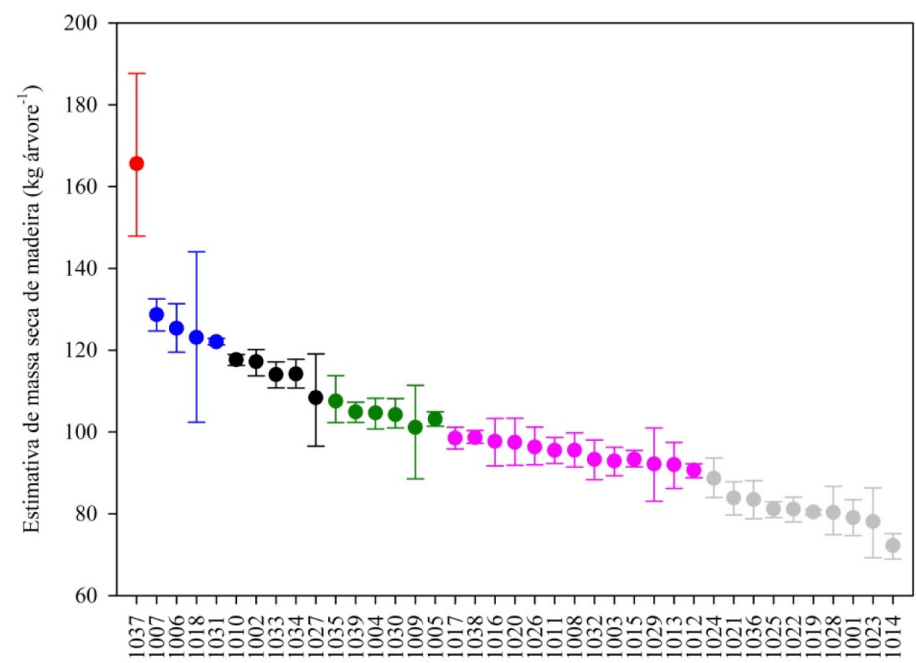

Figura 5. Estimativa de massa seca de madeira das árvores dos clones de Eucalyptus. As cores representam os grupos formados pelo teste de $\operatorname{Scott-Knott~}(a=0,05)$, as barras referem-se aos valores de máximo e mínimo e o coeficiente de variação experimental foi de $7,0 \%$.

Figure 5. Estimates of wood dry matter of trees of Eucalyptus clones. The colors represent the groups formed by the Scott-Knott test $(a=0.05)$, the bars refer to maximum and minimum values and the experimental coefficient of variation was $7.0 \%$. 


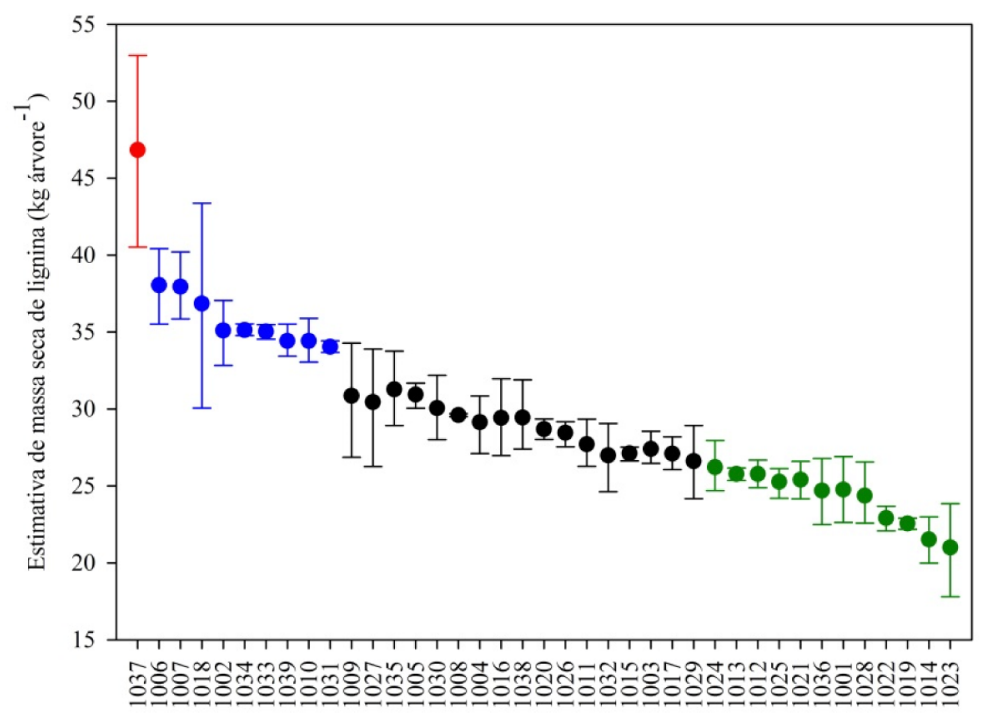

Figura 6. Estimativa de massa seca de lignina das árvores dos clones de Eucalyptus. As cores representam os grupos formados pelo teste de Scott-Knott $(a=0,05)$, as barras referem-se aos valores de máximo e mínimo e o coeficiente de variação experimental foi de $8,0 \%$.

Figure 6. Estimates of lignin dry matter of trees of Eucalyptus clones. The colors represent the groups formed by the Scott-Knott test $(a=0.05)$, the bars refer to maximum and minimum values and the experimental coefficient of variation was $8.0 \%$.

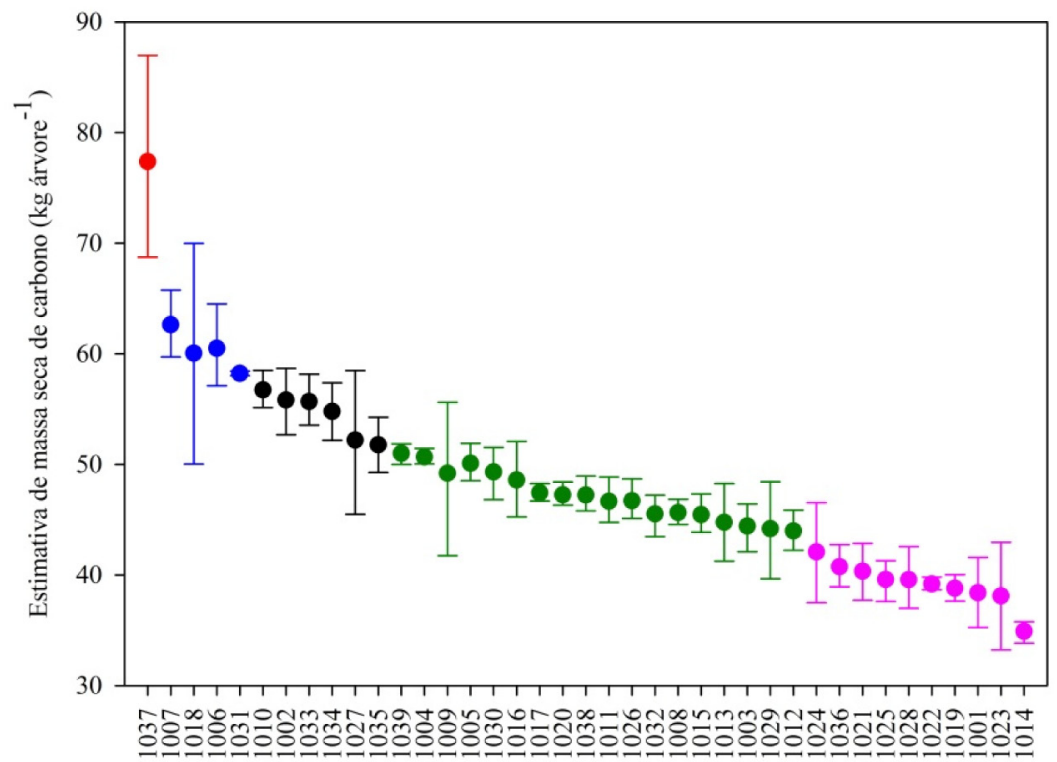

Figura 7. Estimativa de massa seca de carbono das árvores dos clones de Eucalyptus. As cores representam os grupos formados pelo teste de Scott-Knott $(a=0,05)$, as barras referem-se aos valores de máximo e mínimo e o coeficiente de variação experimental foi de 7,5\%.

Figure 7. Estimates of carbon dry matter of trees of Eucalyptus clones. The colors represent the groups formed by the Scott-Knott test $(a=0.05)$, the bars refer to maximum and minimum values and the experimental coefficient of variation was $7.5 \%$.

A alteração no ranqueamento dos clones demonstra a importância de considerar as estimativas de massa seca na classificação de clones de Eucalyptus para a geração de calor ou produção de carvão vegetal. O clone 1037 foi superior em aproximadamente 94\% aos clones classificados nos grupos com os menores valores de massas secas de lignina e carbono por árvore.

\section{Análise de agrupamento (cluster)}

Na Figura 8 encontra-se o dendrograma do agrupamento dos clones de Eucalyptus avaliados. O coeficiente de correlação cofenética encontrado foi de 0,674 e significativo a 1\% de significância pelo teste t. Dessa forma, o dendrograma obtido pode ser considerado consistente e inferências de interesse com base na sua avaliação visual podem ser realizadas. Resultado similar foi relatado por Couto et al. (2013) para a análise de agrupamento de clones de Eucalyptus grandis e Eucalyptus 
Protásio et al. - Classificação de clones de Eucalyptus por meio da relação

Siringil/Guaiacil e das características de crescimento para uso energético

urophylla. Os autores observaram coeficiente de correlação cofenética de 0,68 utilizando a mesma metodologia deste trabalho.

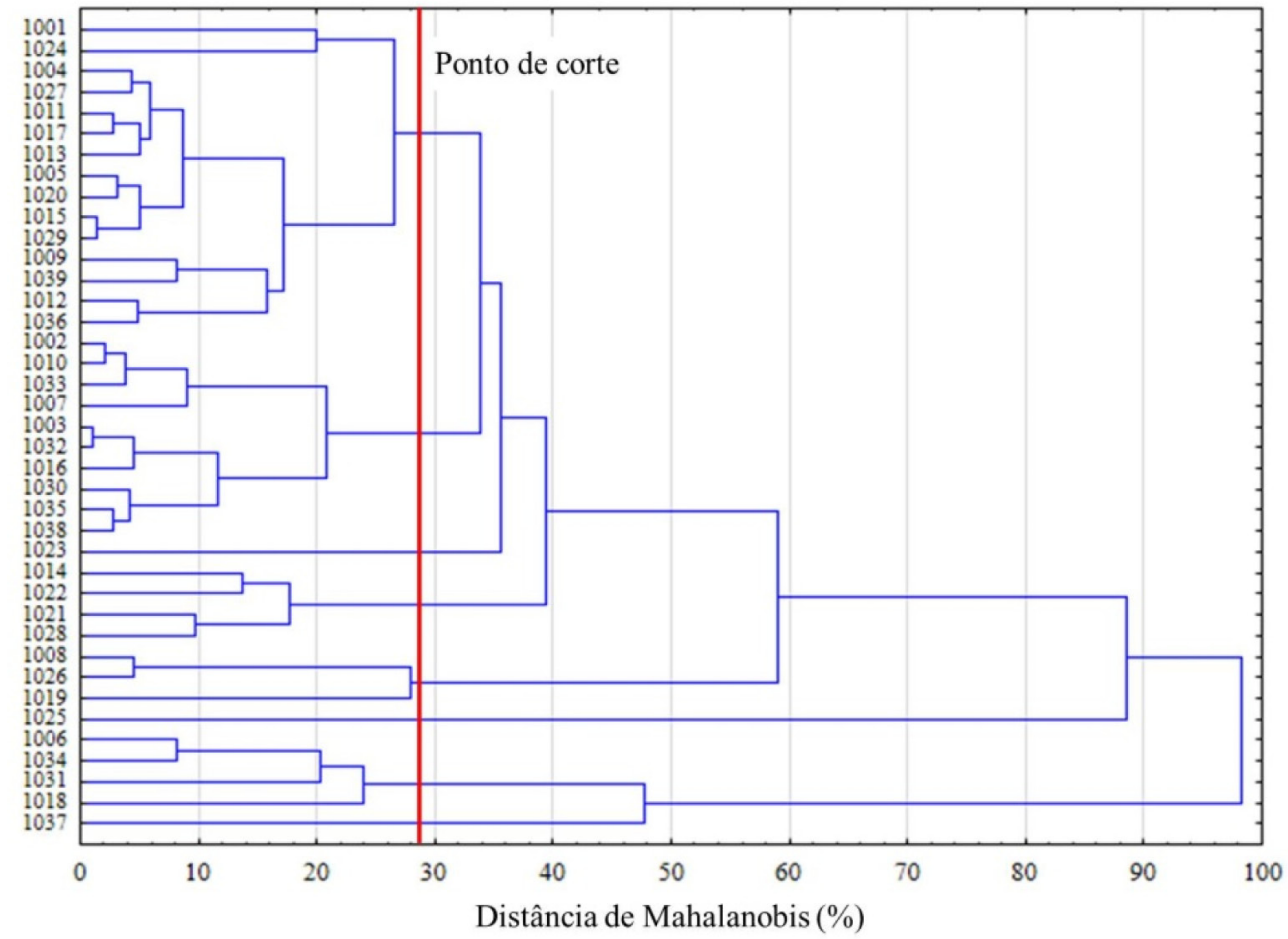

Figura 8. Dendrograma do agrupamento dos 39 clones de Eucalyptus avaliados.

Figure 8. Grouping dendrogram of the 39 Eucalyptus clones evaluated.

Pelo ponto de corte estabelecido, equivalente a 28,4\% da distância total, observou-se a formação de oito grupos distintos (Tabela 2). Pela análise das distâncias entre os indivíduos constatou-se que os clones 1025 (Híbrido de Eucalyptus camaldulensis) e 1037 (Eucalyptus sp.) são os mais divergentes, enquanto os clones 1003 (Eucalyptus urophylla) e 1032 (Eucalyptus sp.) podem ser considerados os mais similares.

Analisando-se as distâncias médias de Mahalanobis intra e intergrupo (Tabela 3), observa-se a similaridade dos grupos I e II, I e IV e II e V, demonstrando que o cruzamento de plantas destes pares de grupos não é indicado se o efeito de heterose for o objetivo. As maiores distâncias foram observadas entre os pares de grupos: III e VIII, IV e VIII e VI e VIII, ou seja, estes são os mais divergentes em relação às características avaliadas (Tabela 4). Cruzamentos de genótipos destes grupos são indicados para aumentar a variabilidade e a expressão do efeito heterótico.

Observou-se que as estimativas de massas secas de madeira e de carbono foram as variáveis que mais contribuíram para a diversidade genética entre os indivíduos com importância relativa de 28,84\% e 26,36\%, respectivamente. Todavia, o volume individual sem casca, densidade básica, teor de lignina total, relação S/G e estimativa de massa seca de lignina contribuíram com 15,04\%, $16,57 \%, 6,57 \%, 4,15 \%$ e 2,48\%, respectivamente. Esses resultados reforçam as tendências observadas pelas análises univariadas e evidencia a importância de se considerar a produtividade de massa seca de madeira na classificação de clones para a finalidade energética.

Protásio et al. (2013a) relataram que o teor de lignina total apresentou grande importância no agrupamento dos clones considerando-se as análises de componentes principais e de variáveis canônicas. Castro et al. (2013) mencionaram que a relação siringil/guaiacil (S/G), juntamente com os valores de lignina total, teve grande influência no ranqueamento de clones comerciais de Eucalyptus para a produção de carvão vegetal. Destaca-se que esses autores não utilizaram as características de crescimento, juntamente com a qualidade da madeira, para a classificação dos clones de Eucalyptus para o uso energético. 
Tabela 2. Grupos formados pela análise de agrupamento (cluster) considerando o método da ligação média (UPGMA).

Table 2. Groups formed by cluster analysis, considering the average-linkage method (UPGMA).

\begin{tabular}{ll}
\hline Grupos & Clones \\
\hline I & $1001,1004,1005,1009,1011,1012,1013,1015,1017,1020,1024,1027,1029,1036$ e 1039 \\
II & $1002,1003,1007,1010,1016,1030,1032,1033,1035$ e 1038 \\
III & 1023 \\
IV & $1014,1021,1022$ e 1028 \\
V & 1008,1019 e 1026 \\
VI & 1025 \\
VII & $1006,1018,1031$ e 1034 \\
VIII & 1037 \\
\hline
\end{tabular}

Tabela 3. Distâncias médias de Mahalanobis entre e dentro dos grupos obtidos pela análise de cluster.

Table 3. Average Mahalanobis distance between and within groups obtained by cluster analysis.

\begin{tabular}{|c|c|c|c|c|c|c|c|c|}
\hline Grupo & $I$ & II & III & IV & $\mathbf{V}$ & VI & VII & VIII \\
\hline$I$ & 15,8 & & & & & & & \\
\hline II & 18,3 & 14,7 & & & & & & \\
\hline III & 100,8 & 113,7 & 0,0 & & & & & \\
\hline IV & 30,9 & 41,2 & 80,0 & 15,7 & & & & \\
\hline V & 83,8 & 35,5 & 162,0 & 64,5 & 20,1 & & & \\
\hline VI & 37,5 & 82,6 & 221,6 & 87,8 & 157,8 & 0,0 & & \\
\hline VII & 164,1 & 89,2 & 308,1 & 211,6 & 60,0 & 225,3 & 20,2 & \\
\hline VIII & 233,4 & 145,5 & 444,1 & 319,8 & 151,1 & 305,3 & 44,6 & 0,0 \\
\hline
\end{tabular}

Além disso, mesmo apresentando baixa contribuição na diversidade genética dos clones de Eucalyptus, o teor de lignina total, a relação S/G e a massa seca de lignina diferiram estatisticamente entre os grupos formados (Tabela 4). Portanto, são parâmetros que não devem ser negligenciados em programas de melhoramento genético visando à obtenção de indivíduos mais adequados para a produção direta de energia (combustão) e para a atividade de carvoejamento.

Tabela 4. Valores médios das variáveis analisadas em cada grupo formado pela análise de cluster.

Table 4. Average values of the variables analyzed in each group formed by cluster analysis.

\begin{tabular}{|c|c|c|c|c|c|c|c|}
\hline Grupo & $\mathrm{DB}\left(\mathrm{kg} \mathrm{m}^{-3}\right)$ & LT (\%) & $\mathbf{S} / \mathbf{G}$ & $\begin{array}{c}\text { Vsc } \\
\left(\mathrm{m}^{3} \text { árvore }^{-1}\right) \\
\end{array}$ & MSM & $\frac{\text { MSL }}{\left.\text { (kg árvore }^{-1}\right)}$ & MSC \\
\hline I & $549 b$ & $29,4 \mathrm{a}$ & $3,1 \mathrm{~b}$ & $0,1743 \mathrm{e}$ & $95,8 \mathrm{~d}$ & $28,1 \mathrm{~d}$ & $46,3 d$ \\
\hline II & $521 \mathrm{c}$ & 29,7 a & $3,2 b$ & $0,2052 \mathrm{c}$ & $107,2 \mathrm{c}$ & $31,9 \mathrm{c}$ & $51,7 \mathrm{c}$ \\
\hline III & $511 \mathrm{c}$ & $26,1 \mathrm{c}$ & $3,7 \mathrm{a}$ & $0,1508 \mathrm{f}$ & $76,8 \mathrm{f}$ & $20,0 \mathrm{~g}$ & $37,6 \mathrm{f}$ \\
\hline IV & $499 d$ & 29,4 a & $2,7 \mathrm{c}$ & $0,1511 \mathrm{f}$ & $74,9 \mathrm{f}$ & $22,1 \mathrm{f}$ & $36,4 f$ \\
\hline V & $458 \mathrm{f}$ & 29,7 a & $3,0 \mathrm{~b}$ & $0,1900 \mathrm{~d}$ & $86,3 \mathrm{e}$ & $25,7 \mathrm{e}$ & $41,6 \mathrm{e}$ \\
\hline VI & 564 a & 30,3 a & $2,5 \mathrm{c}$ & $0,1415 \mathrm{~g}$ & $79,6 \mathrm{f}$ & $24,1 \mathrm{e}$ & $39,3 \mathrm{e}$ \\
\hline VII & $485 \mathrm{e}$ & 29,7 a & $3,1 \mathrm{~b}$ & $0,2403 \mathrm{~b}$ & 116,5 b & $34,7 \mathrm{~b}$ & $56,2 \mathrm{~b}$ \\
\hline VIII & $514 c$ & $28,3 \mathrm{~b}$ & $3,3 \mathrm{~b}$ & $0,3080 \mathrm{a}$ & $159,4 \mathrm{a}$ & $45,6 \mathrm{a}$ & $75,9 a$ \\
\hline
\end{tabular}

DB: densidade básica da madeira; LT: teor de lignina total; S/G: relação siringil/guaiacil; Vsc: volume individual sem casca; MSM, MSL e MSC: estimativas de massa seca de madeira, lignina total e carbono. Médias seguidas pela mesma letra não diferem entre si pelo teste de Scott-Knott a 5\% de significância.

O grupo VIII, formado pelo clone 1037, apesar de apresentar valores intermediários para a densidade básica da madeira, teor de lignina total e relação S/G é o mais indicado para a geração de calor ou para a produção de carvão vegetal, pois apresentou os melhores resultados para a produtividade volumétrica e as estimativas de massas secas de madeira, carbono e lignina por árvore.

O grupo VI, representado pelo clone 1025, apesar dos melhores índices de qualidade da madeira para a finalidade energética, não apresentou desempenho satisfatório quanto ao crescimento e produtividade de massa seca. Esses resultados corroboram com os agrupamentos univariados discutidos anteriormente.

\section{CONCLUSÕES}

As estimativas de massa seca de madeira e massa seca de carbono foram as variáveis que mais contribuíram para a divergência genética total e, portanto, são decisivas no agrupamento e classificação dos clones de Eucalyptus para a finalidade energética. 
O teor de lignina total, a relação S/G e a estimativa de massa seca de lignina contribuíram pouco para a diversidade genética dos materiais analisados, mas não devem ser negligenciadas no ranqueamento de clones para a produção de carvão vegetal ou energia térmica.

O clone 1037 pode ser considerado o mais indicado para a produção de carvão vegetal ou geração direta de energia, pois apresentou melhores resultados para a produtividade de massa seca e mostrou-se dissimilar em relação aos demais por meio das técnicas estatísticas utilizadas.

Novos estudos devem ser realizados visando correlacionar a relação siringil/guaiacil com a combustibilidade da madeira de Eucalyptus e com a produção e qualidade do carvão vegetal produzido subsidiando, assim, os programas de melhoramento genético.

\section{AGRADECIMENTOS}

Ao CNPq, pela concessão das bolsas de mestrado (processo 132431/2013-0) e de doutorado (processo 141439/2014-9) do primeiro autor.

À empresa Plantar, pela doação do material biológico utilizado e auxílio financeiro.

À equipe do Laboratório Multiusuário de Biomateriais da Universidade Federal de Lavras.

\section{REFERÊNCIAS BIBLIOGRÁFICAS}

ABNT - ASSOCIAÇÃO BRASILEIRA DE NORMAS TÉCNICAS. NBR 11941: Madeira - Determinação da densidade básica. Rio de Janeiro, 2003. 6 p.

ALVARES, R. C.; REIS, E. F.; PINTO, J. F. N. Genetic divergence in pepper genotypes from southwest Goiás. Ciência e Agrotecnologia, Lavras, v. 36, n. 5, p. 498-506, 2012.

ARANTES, M. D. C.; TRUGILHO, P. F.; SILVA, J. R. M.; ANDRADE, C. R. Características do carvão de um clone de Eucalyptus grandis W. Hill ex Maiden x Eucalyptus urophylla S. T. Blake. Cerne, Lavras, v.19, n.3, p.423-431, 2013.

ARANTES, M. D. C.; TRUGILhO, P. F.; LiMA, J. T.; CARNEIRO, A. C. O.; ALVES, E.; GUERREIRO, M. C. Longitudinal and radial variation of extractives and total lignin contents in a clone of Eucalyptus grandis W. Hill ex Maiden x Eucalyptus urophylla S. T. Blake. Cerne, Lavras, v.17, n.3, p.283-291, 2011.

ARAÚJO, A. C.; TRUGILHO, P. F.; NAPOLI, A.; BRAGA, P. P. C.; LIMA, R. V.; PROTÁSIO, T. P. Efeito da relação siringil/guaiacil e de fenóis derivados da lignina nas características da madeira e do carvão vegetal de Eucalyptus spp. Scientia Forestalis, Piracicaba, v. 44, n. 110, p. 405-414, 2016.

BOTREL, M. C. G.; TRUGILHO, P. F.; ROSADO, S. C. S.; SILVA, J. R. M. Seleção de clones de Eucalyptus para biomassa florestal e qualidade da madeira. Scientia Forestalis, Piracicaba, v. 38, n. 86, p. 237-245, 2010.

CARNEIRO, A. C. O.; CASTRO, A. F. M.; CASTRO, R. V. O.; SANTOS, R. C.; FERREIRA, L. P.; DAMÁSIO, R. A. P.; VITAL, B. R. Potencial energético da madeira de Eucalyptus sp. em função da idade e de diferentes materiais genéticos. Revista Árvore, Viçosa, v.38, n.2, p.375-381, 2014.

CASTRO, A. F. N. M.; CASTRO, R. V. O.; CARNEIRO, A. C. O.; SANTOS, R. C.; CARVALHO, A. M. L.; TRUGILHO, P. F.; MELO, I. C. N. A. Correlations between age, wood quality and charcoal quality of Eucalyptus clones. Revista Árvore, Viçosa, v.40, n.3, p.551-560, 2016.

CASTRO, A. F. N. M.; CASTRO, R. V. O.; CARNEIRO, A. C. O.; LIMA, J. E.; SANTOS, R. C.; PEREIRA, B. L. C.; ALVES, I. C. N. Análise multivariada para seleção de clones de eucalipto destinados à produção de carvão vegetal. Pesquisa Agropecuária Brasileira, Brasília, v. 48, n. 6, p.627-635, 2013.

COUTO, A. M.; TRUGILHO, P. F.; NAPOLI, A.; LIMA, J. T.; SILVA, J. R. M.; PROTÁSIO, T. P. Qualidade do carvão vegetal de Eucalyptus e Corymbia produzido em diferentes temperaturas finais de carbonização. Scientia Forestalis, Piracicaba, v. 43, n. 108, p. 817-831, 2015. 
COUTO, A. M.; PROTÁSIO, T. P.; TRUGILHO, P. F.; NEVES, T. A.; SÁ, V. A. Multivariate analysis applied to evaluation of Eucalyptus clones for bioenergy production. Cerne, Lavras, v. 19, n. 4, p. 525-533, 2013.

CRUZ, C. D. GENES - a software package for analysis in experimental statistics and quantitative genetics. Acta Scientiarum, Maringá, v.35, n.3, p.271-276, 2013.

CRUZ, C. D.; CARNEIRO, P.C.S. Modelos biométricos aplicados ao melhoramento genético. Viçosa: UFV, 2006. 585 p.

DOWNES, G. M.; HUDSON, I. L.; RAYMOND, C. A.; DEAN, G. H.; MICHELL, A. J.; SCHIMLECK, R.; EVANS, R.; MUNERI, A. Sampling plantation eucalypts for wood and fiber properties. Collingwood: CSIRO. Melbourne: CSRIO, 1997. 126 p.

EPE - EMPRESA DE PESQUISA ENERGÉTICA. Balanço Energético Nacional 2015: ano base 2014. Rio de Janeiro: EPE, 2015. 292 p.

GOLDSCHIMID, O. Ultraviolet spectra. In: SARKANEN, K.V.; Ludwig, C.H. (Ed.). Lignins: occurrence, formation, structure and reactions. New York: J. Wiley, 1971. p.241-298.

GOMIDE, J. L.; DEMUNER, B. J. Determinação do teor de lignina em material lenhoso: método Klason modificado. O Papel, São Paulo, v. 47, n. 8, p. 36-38, 1986.

GOUVÊA, A. F. G; TRUGILHO, P. F.; ASSIS, C. O.; ASSIS, M. R.; COLODETTE, J. L.; GOMES, C. M. Avaliação do efeito da relação siringila/guaiacila da lignina de eucalipto na produção de carvão vegetal. Ciência da Madeira, Pelotas, v. 6, n. 2, p.71-78, 2015.

HAIR JÚNIOR, J. F.; BLACK, W. C.; BABIN, B. J.; ANDERSON, R. E.; TATHAM, R. L. Análise multivariada de dados. Porto Alegre: Bookman, 2009. 688 p.

IBÁ - INDÚSTRIA BRASILEIRA DE ÁRVORES. Relatório IBÁ 2015. Brasília: IBÁ, 2015. 64 p.

LIN, S. Y.; DENCE, C. W. Methods in lignin chemistry. Berlin: Springer-Verlag, 1992. 578 p.

MENESES, V. A.; TRUGILHO, P. F.; CALEGARIO, N.; LEITE, H. G. Efeito da idade e do sítio na densidade básica e produção de massa seca de madeira em um clone do Eucalyptus urophylla. Scientia Forestalis, Piracicaba, v. 43, n. 105, p. 101-116, 2015.

MILLIGAN, G. W.; COOPER, M. C. An examination of procedures for determining the number of clusters in a data set. Psychometrika, Madison, v. 50, n. 2, p. 159-179, 1985.

MOJENA, R. Hierarchical grouping methods and stopping rules: an valuation. The Computer Journal, Oxford, v. 20, n. 4, p. 359-363, 1977.

MUNIR, S.; DAOOD, S. S.; NIMMO, W.; CUNLIFFE, A. M.; GIBBS, B. M. Thermal analysis and devolatilization kinetics of cotton stalk, sugar cane bagasse and shea meal under nitrogen and air atmospheres. Bioresource Technology, London, v. 100, n.3, p. 1413-1418, 2009.

NEVES, T. A.; PROTÁSIO, T. P.; TRUGIlHO, P. F.; VAlLE, M. L. A.; SOUSA, L. C.; VIEIRA, C. M. Qualidade da madeira de clones de Eucalyptus em diferentes idades para a produção de bioenergia. Revista de Ciências Agrárias, Belém, v. 56, n. 2, p.139-148, 2013.

NEVES, T. A.; PROTÁSIO, T. P.; COUTO, A. M.; TRUGILHO, P. F.; SILVA, V. O.; VIEIRA, C. M. M. Avaliação de clones de Eucalyptus em diferentes locais visando à produção de carvão vegetal. Pesquisa Florestal Brasileira, Colombo, v. 31, n. 68, p. 319 - 330, 2011.

Sci. For., Piracicaba, v. 45, n. 114, p. 327-341, jun. 2017 DOI: dx.doi.org/10.18671/scifor.v45n114.09 
Protásio et al. - Classificação de clones de Eucalyptus por meio da relação

Siringil/Guaiacil e das características de crescimento para uso energético

OliveirA, A. C.; ROCHA, M. F. V.; PEREIRA, B. L. C.; CARNEIRO, A. C. O.; CARVAlHO, A. M. M. L.; VITAL. B. R. Avaliação de diferentes níveis de desbaste nas propriedades da madeira e do carvão vegetal de Eucalyptus grandis x Eucalyptus urophylla. Floresta, Curitiba, v. 42, n. 1, p. 59 - 68, 2012.

PEREIRA, B. L. C.; CARneiro, A. C. O.; CARVAlhO, A. M. M. L.; COlODETTE, J. L.; OliveIRA, A. C.; FONTES, M. P. F. Influence of chemical composition of Eucalyptus wood on gravimetric yield and charcoal properties. Bioresources, Railegh, v. 8, p. 4574-4592, 2013.

PEREIRA, B. L. C.; OliveIRA, A. C.; CARVAlHO, A. M. M. L.; CARNEIRO, A. C. O.; SANTOS, L. C.; VITAL, B. R. Quality of wood and charcoal from Eucalyptus clones for ironmaster use. International Journal of Forestry Research, New York, v. 2012, p. 1-8, 2012.

PROTÁSIO, T. P.; COUTO, A. M.; TRUGILHO, P. F.; GUIMARÃES JUNIOR, J. B.; LIMA JUNIOR, P. H.; SILVA, M. M. O. Avaliação tecnológica do carvão vegetal da madeira de clones jovens de Eucalyptus grandis e Eucalyptus urophylla. Scientia Forestalis, Piracicaba, v. 43, n. 108, p. 801-816, 2015.

PROTÁSIO, T. P.; COUTO, A. M.; REIS, A. A.; TRUGILHO, P. F. Seleção de clones de Eucalyptus para a produção de carvão vegetal e bioenergia por meio de técnicas univariadas e multivariadas. Scientia Forestalis, Piracicaba, v. 42, n. 97, 2013a.

PROTÁSIO, T. P.; COUTO, A. M.; REIS, A. A.; TRUGIlHO, P. F.; GODINHO, T. P. Potencial siderúrgico e energético do carvão vegetal de clones de Eucalyptus spp. aos 42 meses de idade. Pesquisa Florestal Brasileira, Colombo, v. 33, n. 74, p. 137-149, 2013b.

R CORE TEAM. R: A language and environment for statistical computing. 2014. Disponível em: < http:// www.R-project.org/ >. Acesso em: 6 abr. de 2016.

RAAD, T. J.; PINHEIRO, P. C. C.; YOSHIDA, M. I. Equação geral de mecanismos cinéticos da carbonização do Eucalyptus spp. Cerne, Lavras, v. 12, n. 2, p. 93-106, 2006.

REIS, A. A.; MElO, I. C. N. A.; PROTÁSIO, T. P.; TRUGILHO, P. F.; CARNEIRO, A. C. Efeito de local e espaçamento na qualidade do carvão vegetal de um clone de Eucalyptus urophylla S. T. Blake. Floresta e Ambiente, Seropédica, v. 19, n. 4, p.497-505, 2012.

SANTANA, W. M. S.; CALEGARIO, N.; ARANTES, M. D. C.; TRUGILHO, P. F. Effect of age and diameter class on the properties of wood from clonal Eucalyptus. Cerne, Lavras, v.18, n.1, p.1-8, 2012.

SANTOS, R. C.; CARNEIRO, A. C. O.; VITAL, B. R.; CASTRO, R. V. O.; VIDAURRE, G. B.; TRUGILHO, P. F.; CASTRO, A. F. M. Influência das propriedades químicas e da relação siringil/guaiacil da madeira de eucalipto na produção de carvão vegetal. Ciência Florestal, Santa Maria, v. 26, n. 2, p. 657-669, 2016.

SARKANEN, K.V.; LUDWIG, C.H. Definition and Nomenclature. In: SARKANEN; SUDWIG. Lignins: occurrence, formation, structure and reaction. New York: John Wiley \& Sons, 1971. 916 p.

SCOTT, A. J.; KNOTT, M. A cluster analysis method for grouping means in the analysis of variance. Biometrics, Washington, v. 30, n. 2 p. 507-512, 1974.

SHEN, J.; ZHU, S.; LIU, X.; ZHANG, H.; TAN, J. The prediction of elemental composition of biomass based on proximate analysis. Energy Conversion and Management, Oxford, v. 51, n. 5, p. 983-987, 2010.

SINGH, D. The relative importance of characters affecting genetic divergence. Indian Journal of Genetics and Plant Breeding, New Delhi, v. 41, n. 2, p. 237-245, 1981. 
SOARES, V. C.; BIANCHI, M. L.; TRUGILHO, P. F.; HÖFLER, J.; PEREIRA, A. J. Análise das propriedades da madeira e do carvão vegetal de híbridos de eucalipto em três idades. Cerne, Lavras, v. 21, n. 2, p. 191-197, 2015.

SOARES, V. C.; BIANCHI, M. L.; TRUGILHO, P. F.; PEREIRA, A. J.; HÖFLER, J. Correlações entre as propriedades da madeira e do carvão vegetal de híbridos de eucalipto. Revista Árvore, Viçosa, v.38, n.3, p.543-549, 2014.

SOKAL, R. R.; ROHLF, F. J. The comparison of dendrograms by objective methods. Taxon, Berlin, v. 11, n. 2, p.33-40, 1962.

TELMO, C.; LOUSADA, J. Heating values of wood pellets from different species. Biomass and Bioenergy, Oxford, v.35, n.7, p. 2634-2639, 2011.

TRUGILHO, P. F. Densidade básica e estimativa de massa seca e de lignina na madeira em espécies de Eucalyptus. Ciência e Agrotecnologia, Lavras, v. 33, n. 5, p. 1228-1239, 2009.

TRUGILHO, P. F.; GOULART, S. L.; ASSIS, C. O.; COUTO, F. B. S.; ALVES, I. C. N.; PROTÁSIO, T. P.; NAPOLI, A. Características de crescimento, composição química, física e estimativa de massa seca de madeira em clones e espécies de Eucalyptus jovens. Ciência Rural, Santa Maria, v.45, n.4, p.661-666, 2015.

TRUGIlHO, P. F.; ARANTES, M. D. C.; PÁDUA, F. A.; ALMADO, R. P.; BALIZA, A. E. R. Estimativa de carbono fixado na madeira de um clone híbrido de Eucalyptus urophylla e Eucalyptus grandis. Cerne, Lavras, v.16, p.3340, 2010.

TRUGILHO, P. F.; LIMA, J. T.; MORI, F. A.; LINO, A. L. Avaliação de clones de Eucalyptus para a produção de carvão vegetal. Cerne, Lavras, v. 7, n.2, p.104-114, 2001.

YANG, H.; YAN, R.; CHEN, H.; LEE, D. H.; ZHENG, C. Characteristics of hemicellulose, cellulose and lignin pyrolysis. Fuel, London, v.86, n. 12-13, p. 1781-1788, 2007.

YIN, C. Y. Prediction of higher heating values of biomass from proximate and ultimate analyses. Fuel, London, v.90, n. 3, p. 1.128-1.132, 2011.

Recebido em 05/05/2016

Aceito para publicação em 17/01/2017

Sci. For., Piracicaba, v. 45, n. 114, p. 327-341, jun. 2017

DOI: dx.doi.org/10.18671/scifor.v45n114.09 
